\title{
GRAPH AND DEPTH OF A MONOMIAL SQUAREFREE IDEAL
}

\author{
DORIN POPESCU
}

(Communicated by Irena Peeva)

\begin{abstract}
Let $I$ be a monomial squarefree ideal of a polynomial ring $S$ over a field $K$ such that the sum of every three different ideals of its minimal prime ideals is the maximal ideal of $S$, or more generally a constant ideal. We associate to $I$ a graph on $[s], s=|\operatorname{Min} S / I|$, on which we may read the depth of $I$. In particular, depth $I$ does not depend on char $K$. Also we show that $I$ satisfies Stanley's Conjecture.
\end{abstract}

\section{INTRODUCTION}

Let $S=K\left[x_{1}, \ldots, x_{n}\right], n \in \mathbf{N}$, be a polynomial ring over a field $K$, and $I \subset S$ a monomial squarefree ideal with minimal prime ideals $P_{1}, \ldots, P_{s}$ (here we study only the monomial squarefree ideals). According to 4 the size of $I$ is the number $v+(n-h)-1$, where $h$ is the height of $\sum_{j=1}^{s} P_{j}$ and $v$ is the minimal number $e$ for which there exist integers $i_{1}<i_{2}<\cdots<i_{e}$ such that $\sum_{k=1}^{e} P_{i_{k}}=\sum_{j=1}^{s} P_{j}$. Similarly, we defined in [8] the bigsize of $I$, which is the number $t+(n-h)-1$, where $t$ is the minimal number $e$ such that for all integers $i_{1}<i_{2}<\cdots<i_{e}$ it follows that $\sum_{k=1}^{e} P_{i_{k}}=\sum_{j=1}^{s} P_{j}$. Clearly bigsize $(I) \geq \operatorname{size}(I)$. Lyubeznik [4] showed that depth $I \geq 1+\operatorname{size} I$.

Let $I \subset S$ be a monomial ideal of $S, u \in I$ a monomial and $u K[Z], Z \subset$ $\left\{x_{1}, \ldots, x_{n}\right\}$ the linear $K$-subspace of $I$ of all elements $u f, f \in K[Z]$. A presentation of $I$ as a finite direct sum of such spaces $\mathcal{D}: I=\bigoplus_{i=1}^{r} u_{i} K\left[Z_{i}\right]$ is called a Stanley decomposition of $I$. Set $\operatorname{sdepth}(\mathcal{D})=\min \left\{\left|Z_{i}\right|: i=1, \ldots, r\right\}$ and

sdepth $I:=\max \{\operatorname{sdepth}(\mathcal{D}): \mathcal{D}$ is a Stanley decomposition of $I\}$.

The Stanley Conjecture 11 says that sdepth $I \geq \operatorname{depth} I$. This conjecture holds for arbitrary monomial squarefree ideals if $n \leq 5$ by [7] (see especially the arXiv version), or for intersections of four monomial prime ideals by [5], [8. In the case of nonsquarefree monomial ideals $J$, an important inequality is $\operatorname{sdepth} J \leq \operatorname{sdepth} \sqrt{J}$ (see [3, Theorem 2.1]). Similarly to Lyubeznik's result, sdepth $I \geq 1+\operatorname{size} I$ by [2, Theorem 3.1]. If bigsize $(I)=$ size $(I)$, then $I$ satisfies Stanley's Conjecture by [2, Theorems 1.2, 3.1].

The purpose of this paper is to study the case when $\operatorname{bigsize}(I)=2$, $\operatorname{size}(I)=1$. In the case $\sum_{j=1}^{s} P_{j}=m=\left(x_{1}, \ldots, x_{n}\right)$, we associate to $I$ a graph $\Gamma$ on $[s]$ given

Received by the editors May 6, 2011.

2010 Mathematics Subject Classification. Primary 13C15; Secondary 13F20, 05E40, 13F55, $05 \mathrm{C} 25$.

Key words and phrases. Monomial ideals, join graphs, size, depth, Stanley depth.

The support from CNCSIS grant PN II-542/2009 of the Romanian Ministry of Education, Research and Innovation is gratefully acknowledged. 
by $\{i j\}$ is an edge if and only if $P_{i}+P_{j}=m$. We express the depth of $I$ in terms of the properties of $\Gamma$ and of $q(I)=\min \left\{\operatorname{dim} S /\left(P_{i}+P_{j}\right): j \neq i, P_{i}+P_{j} \neq m\right\}$. We note that [8, Lemmas 3.2,3.4] say, in particular, that $\operatorname{depth}_{S} I=2$ if and only if $\Gamma$ is a join graph. Our Corollary 2.8 says that if $q(I)>1$, then $\operatorname{depth}_{S} I=2+q(I)$ if and only if $\Gamma$ is a so-called concatenation of several graphs on two vertices having no edges. Thus knowing $q(I) \operatorname{depth}_{S} I$ can be read on $\Gamma$ (see Corollary 2.9) ). It follows that for a monomial squarefree ideal $I \subset S$ such that the sum of every three different of its minimal prime ideals is a constant ideal (for example $m$ ), $\operatorname{depth}_{S} I$ does not depend of char $K$ (see Theorem 2.10) and Stanley's Conjecture holds (see Theorem 3.5.

It is well known that $\operatorname{depth}_{S} I$ depends on the characteristic of $K$ if $\operatorname{bigsize}(I)=$ $5, \operatorname{size}(I)=2$ (see our Remark 2.11), so it is very likely that this case is much harder for proving Stanley's Conjecture. Several people ask if there exist examples when the special Stanley decomposition of [5, 8 , or the splitting variables in the terminology of 2] do not help in proving Stanley's Conjecture since there exists no good main prime ideal. Our Example 3.3 is such an example.

\section{Depth two And Three}

Let $S=K\left[x_{1}, \ldots, x_{n}\right], n \in \mathbf{N}$ be a polynomial ring over a field $K$ and $\tilde{S}=$ $K\left[x_{1}, \ldots, x_{n-1}\right] \subset S$. We start by recalling the following two lemmas from [7].

Lemma 1.1. Let $I, J \subset \tilde{S}, I \subset J, I \neq J$ be two monomial ideals, $T=\left(I+x_{n} J\right) S$ such that

(1) $\operatorname{depth}_{\tilde{S}} \tilde{S} / I \neq \operatorname{depth}_{S} S / T-1$,

(2) $\operatorname{sdepth}_{\tilde{S}} I \geq \operatorname{depth}_{\tilde{S}} I, \quad \operatorname{sdepth}_{\tilde{S}} J \geq \operatorname{depth}_{\tilde{S}} J$.

Then $\operatorname{sdepth}_{S} T \geq \operatorname{depth}_{S} T$.

Lemma 1.2. Let $I, J \subset \tilde{S}, I \subset J, I \neq J$ be two monomial ideals, $T=\left(I+x_{n} J\right) S$ such that

(1) $\operatorname{depth}_{\tilde{S}} \tilde{S} / I=\operatorname{depth}_{S} S / T-1$,

(2) $\operatorname{sdepth}_{\tilde{S}} I \geq \operatorname{depth}_{\tilde{S}} I$,

(3) $\operatorname{sdepth}_{\tilde{S}} J / I \geq \operatorname{depth}_{\tilde{S}} J / I$.

Then $\operatorname{sdepth}_{S} T \geq \operatorname{depth}_{S} T$.

The above lemmas allow us to show Stanley's Conjecture in a special case.

Proposition 1.3. Let $T \subset S$ be a monomial squarefree ideal. If $S / T$ is CohenMacaulay of dimension 2, then $\operatorname{sdepth}_{S} T \geq \operatorname{depth}_{S} T$.

Proof. We use induction on $n$, the case $n \leq 5$ being given in [7. Suppose $n>5$. Then $T$ has the form $T=I+x_{n} J$ for two monomial squarefree ideals $I, J \subset \tilde{S}$; in fact, $I=T \cap \tilde{S}, J=\left(T: x_{n}\right) \cap \tilde{S}$. Note that $\operatorname{dim} \tilde{S} / I=\operatorname{dim} S /\left(T, x_{n}\right) \leq 2$

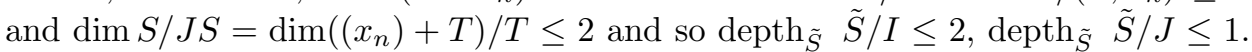
If $\operatorname{depth}_{\tilde{S}} \tilde{S} / I=2$, then $\operatorname{sdepth}_{\tilde{S}} I \geq \operatorname{depth}_{\tilde{S}} I$ by the induction hypothesis. If $\operatorname{depth}_{\tilde{S}} \tilde{S} / I=1$ (by [10, Proposition 1.2] $\operatorname{depth}_{\tilde{S}} \tilde{S} / I>0$ ), then $\operatorname{depth}_{\tilde{S}} I=2=$ $1+\operatorname{size}(I) \leq \operatorname{sdepth}_{\tilde{S}} I$ by [2, Theorem 3.1] and similarly for $J$. As $\operatorname{dim} J / I \leq$ $\operatorname{dim} \tilde{S} / I \leq \operatorname{dim} S / T=2$ we have $\operatorname{sdepth}_{\tilde{S}} J / I \geq \operatorname{depth}_{\tilde{S}} J / I$ by $[6$. Now the result is a consequence of Lemmas 1.1, 1.2 if $I \neq J$; otherwise $T=I S$, and we may apply [2, Lemma 3.6]. 
Let $I=\bigcap_{i=1}^{s} P_{i}, s \geq 3$ be the intersection of the minimal monomial prime ideals of $S / I$. Assume that $\sum_{i=1}^{s} P_{i}=m$ and the bigsize of $I$ is two. Set

$$
q=q(I)=\min \left\{\operatorname{dim} S /\left(P_{i}+P_{j}\right): j \neq i, P_{i}+P_{j} \neq m\right\} .
$$

We will need the following two lemmas from [8].

Lemma 1.4. If $P_{1}+P_{2} \neq m$ and $P_{k}+P_{e}=m$ for all $k, e>2, k \neq e$, then

(1) $\operatorname{depth}_{S} S / I \in\{1,2,1+q\}$,

(2) $\operatorname{depth}_{S} S / I=1$ if and only if there exists $j>2$ such that $P_{1}+P_{j}=m=$ $P_{2}+P_{j}$,

(3) $\operatorname{depth}_{S} S / I>2$ if and only if $q>1$ and each $j>2$ satisfies either

$$
\begin{gathered}
P_{1}+P_{j} \neq m=P_{2}+P_{j} \text { or } \\
P_{2}+P_{j} \neq m=P_{1}+P_{j},
\end{gathered}
$$

(4) $\operatorname{depth}_{S} S / I=2$ if and only if the following conditions hold:

(a) each $j>2$ satisfies either $P_{1}+P_{j} \neq m$ or $P_{2}+P_{j} \neq m$,

(b) $q=1$ or there exists a $k>2$ such that

$$
P_{1}+P_{k} \neq m \neq P_{2}+P_{k},
$$

(5) $\operatorname{sdepth}_{S} I \geq \operatorname{depth}_{S} I$.

Lemma 1.5. Suppose that whenever there exist $i \neq j$ in $[s]$ such that $P_{i}+P_{j} \neq m$, there exist also $k \neq e$ in $[s] \backslash\{i, j\}$ such that $P_{k}+P_{e} \neq m$ (that is, the complementary case of the above lemma). Then

(1) $\operatorname{depth}_{S} S / I \in\{1,2,1+q\}$;

(2) $\operatorname{depth}_{S} S / I=1$ if and only if after a renumbering of $\left\{P_{i}\right\}$ there exists $1 \leq c<s$ such that $P_{i}+P_{j}=m$ for each $c<j \leq s$ and $1 \leq i \leq c$.

These two lemmas allow us to show the following useful proposition.

Proposition 1.6. Suppose that $P_{1}=\left(x_{1}, \ldots, x_{r}\right), 1 \leq r<n, S^{\prime}=K\left[x_{r+1}, \ldots, x_{n}\right]$ and $P_{1}+P_{2} \neq m \neq P_{1}+P_{3}, P_{2}+P_{3} \neq m$. Then depth $h_{S} S / I \leq 2$; in particular,

$$
\operatorname{sdepth}_{S^{\prime}}\left(P_{2} \cap P_{3} \cap S^{\prime}\right) \geq 2 \geq \operatorname{depth}_{S} S / I .
$$

Proof. Apply induction on $s$; the cases $s=3,4$ follow from [5], 8]. Suppose that $s>4$. Set $E=S /\left(P_{1} \cap P_{3} \cap \ldots \cap P_{s}\right) \oplus S /\left(P_{1} \cap P_{2} \cap P_{4} \cap \ldots \cap P_{s}\right)$ and $F=S /\left(P_{1} \cap\left(P_{2}+P_{3}\right) \cap P_{4} \cap \ldots \cap P_{s}\right)$. Note that if $P_{i} \subset P_{2}+P_{3}$ for some $i \neq 2,3$, then $P_{2}+P_{3}=P_{i}+P_{2}+P_{3}=m$ because bigsize of $I$ is two, a contradiction! Thus the bigsize of $F$ is one and so $\operatorname{depth}_{S} S / F=1$ by 8 . From the exact sequence

$$
0 \rightarrow S / I \rightarrow E \rightarrow F \rightarrow 0
$$

we get $\operatorname{depth}_{S} S / I=2$ if $\operatorname{depth}_{S} E>1$. Otherwise, suppose that $G=S /\left(P_{1} \cap P_{2} \cap\right.$ $\left.P_{4} \cap \ldots \cap P_{s}\right)$ has depth one. Then after renumbering $\left\{P_{i}\right\}$ we may suppose that there exists $c \neq 3,1 \leq c<s$, such that $P_{i}+P_{j}=m$ for all $1 \leq i \leq c, c<j \leq s, i, j \neq 3$ (see Lemmas 1.4, 1.5). In fact we may renumber only $\left\{P_{e}\right\}_{e>3}$ and take $c>3$ because $P_{1}+P_{2} \neq m$. Set $M=S / P_{1} \cap \ldots \cap P_{c}$ and $N=M \oplus S / P_{3} \cap P_{c+1} \cap \ldots \cap P_{s}$. In the exact sequence

$$
0 \rightarrow S / I \rightarrow N \rightarrow S / P_{3} \rightarrow 0
$$

we have the depth of all modules $\leq \operatorname{depth}_{S} S / P_{3}$. By the Depth Lemma 12 it follows that $\operatorname{depth}_{S} S / I=\operatorname{depth}_{S} N$ and so $\operatorname{depth}_{S} S / I \leq \operatorname{depth}_{S} M$. Applying the 
induction hypothesis we get $\operatorname{depth}_{S} M \leq 2$, that is, $\operatorname{depth}_{S} S / I \leq 2$. Finally, by 9 ] we have

$$
\begin{gathered}
\operatorname{sdepth}_{S^{\prime}}\left(P_{2} \cap P_{3} \cap S^{\prime}\right) \geq \operatorname{depth}_{S^{\prime}}\left(P_{2} \cap P_{3} \cap S^{\prime}\right)=1+\operatorname{depth}_{S^{\prime}} S^{\prime} /\left(P_{2} \cap P_{3} \cap S^{\prime}\right) \\
=1+\operatorname{depth}_{S} S /\left(P_{1}+P_{2}\right) \cap\left(P_{1}+P_{3}\right)=2
\end{gathered}
$$

because $P_{1}+P_{2}+P_{3}=m$.

Corollary 1.7. Suppose that bigsize $(I)=$ size $(I) \leq 2$. Then $\operatorname{depth}_{S} I$ does not depend on the characteristic of $K$.

Proof. If bigsize $(I)=\operatorname{size}(I)=1$, then $\operatorname{depth}_{S} I=2$ by [8, Corollary 1.7] and so does not depend on the characteristic of $K$. If $\operatorname{bigsize}(I)=\operatorname{size}(I)=2$, then $\operatorname{depth}_{S} I \leq 3$ by the above proposition and so $\operatorname{depth}_{S} I=3$ by [4] independently of char $K$.

Theorem 1.8. If $\operatorname{depth}_{S} I \leq 3$, then $\operatorname{sdepth}_{S} I \geq \operatorname{depth}_{S} I$.

Proof. By [2] we have $\operatorname{sdepth}_{S} I \geq 1+\operatorname{size}(I) \geq 2$, and it is enough to consider the case $\operatorname{depth}_{S} I=3$, that is, $\operatorname{depth}_{S} S / I=2$. If $\operatorname{dim} S / I=2$, then we may apply Proposition 1.3. otherwise we may suppose that $\operatorname{dim} S / P_{i} \geq 3$ for any $i$, let us say $i=1$. We may suppose that $P_{1}=\left(x_{1}, \ldots, x_{r}\right)$ for some $r<n$; thus $n \geq r+3$. Set $S^{\prime \prime}=K\left[x_{1}, \ldots, x_{r}\right], S^{\prime}=K\left[x_{r+1}, \ldots, x_{n}\right]$.

Applying [8, Theorem 1.6] for $\mathcal{F}$ containing some $\tau_{j}=\{j\}$, and $\tau_{j k}=\{j, k\}$, $1<j, k \leq s, j \neq k$, we get $\left.\operatorname{sdepth}_{S} I \geq \min \left\{A_{0},\left\{A_{\tau_{j}}\right\}_{\tau_{j} \in \mathcal{F}}\right\},\left\{A_{\tau_{j k}}\right\}_{\tau_{j k} \in \mathcal{F}}\right\}$ for $A_{0}=\operatorname{sdepth}_{S}\left(I \cap S^{\prime \prime}\right) S$ if $I \cap S^{\prime \prime} \neq 0$ or $A_{0}=n$ otherwise, and

$$
A_{\tau} \geq \operatorname{sdepth}_{S_{\tau}} J_{\tau}+\operatorname{sdepth}_{S^{\prime}} L_{\tau}
$$

where $J_{\tau}=\bigcap_{e \notin \tau} P_{e} \cap S_{\tau} \neq 0, S_{\tau}=K\left[\left\{x_{u}: x_{u} \in S^{\prime \prime}, x_{u} \notin \sum_{e \in \tau} P_{e}\right\}\right], L_{\tau}=$ $\bigcap_{e \in \tau}\left(P_{e} \cap S^{\prime}\right) \neq 0$. If $P_{1}+P_{j} \neq m$, then

$A_{\tau_{j}} \geq \operatorname{sdepth}_{S_{\tau_{j}}} J_{\tau_{j}}+\operatorname{sdepth}_{S^{\prime}}\left(P_{j} \cap S^{\prime}\right) \geq 1+\operatorname{dim} S /\left(P_{1}+P_{j}\right)+\left\lceil\frac{\operatorname{height}\left(P_{j} \cap S^{\prime}\right)}{2}\right\rceil$,

where $\lceil a\rceil, a \in \mathbf{Q}$, denotes the smallest integer $\geq a$. Thus $A_{\tau_{j}} \geq 3=\operatorname{depth}_{S} I$. If $P_{1}+P_{j}=m$, then $P_{j} \cap S^{\prime}$ is the maximal ideal of $S^{\prime}$ and we have

$$
A_{\tau_{j}} \geq 1+\left\lceil\frac{h e i g h t\left(P_{j} \cap S^{\prime}\right)}{2}\right\rceil \geq 1+\left\lceil\frac{3}{2}\right\rceil \geq 3=\operatorname{depth}_{S} I .
$$

If $P_{1}+P_{j} \neq m \neq P_{1}+P_{k}, P_{j}+P_{k} \neq m$, then

$$
A_{\tau_{j k}} \geq \operatorname{sdepth}_{S_{\tau_{j k}}} J_{\tau_{j k}}+\operatorname{sdepth}_{S^{\prime}} L_{\tau_{j k}} \geq 1+\operatorname{sdepth}_{S^{\prime}}\left(P_{j} \cap P_{k} \cap S^{\prime}\right) \geq \operatorname{depth}_{S} I,
$$

by Proposition [1.6. If $P_{1}+P_{j}=m \neq P_{1}+P_{k}, P_{j}+P_{k} \neq m$, then

$$
\operatorname{sdepth}_{S^{\prime}} L_{\tau_{j k}} \geq \operatorname{depth}_{S^{\prime}} L_{\tau_{j k}}=1+\operatorname{dim} S /\left(P_{1}+P_{k}\right) \geq 1+q .
$$

Thus $A_{\tau_{j k}} \geq 2+q \geq 3=\operatorname{depth}_{S} I$. If $P_{1}+P_{j}=m=P_{1}+P_{k}, P_{j}+P_{k} \neq m$, then $L_{\tau_{j k}}$ is the maximal ideal of $S^{\prime}$ and we get $A_{\tau_{j k}} \geq 1+\left\lceil\frac{3}{2}\right\rceil \geq 3=\operatorname{depth}_{S} I$. If $I \cap S^{\prime \prime} \neq 0$, then $A_{0}=\operatorname{sdepth}_{S}\left(I \cap S^{\prime \prime}\right) S \geq 1+n-r \geq \operatorname{depth}_{S} I$. Hence $\operatorname{sdepth}_{S} I \geq \operatorname{depth}_{S} I$. 


\section{Graph of a monomial Squarefree ideal}

Let $I=\bigcap_{i=1}^{s} P_{i}, s \geq 3$ be the intersection of the minimal monomial prime ideals of $S / I$. Assume that $\sum_{i=1}^{s} P_{i}=m$ and the bigsize of $I$ is two. We may suppose that $P_{1}=\left(x_{1}, \ldots, x_{r}\right)$ for some $r<n$ and set

$$
q=q(I)=\min \left\{\operatorname{dim} S /\left(P_{i}+P_{j}\right): j \neq i, P_{i}+P_{j} \neq m\right\} .
$$

Thus $q \leq n-r$. Set $S^{\prime \prime}=K\left[x_{1}, \ldots, x_{r}\right], S^{\prime}=K\left[x_{r+1}, \ldots, x_{n}\right]$.

Definition 2.1. Let $\Gamma$ be the simple graph on $[s]$ given by $\{i j\}$ is an edge (we write $\{i j\} \in E(\Gamma))$ if and only if $P_{i}+P_{j}=m$. We call $\Gamma$ the graph associated to I. $\Gamma$ has the triangle property if there exists $i \in[s]$ such that for all $j, k \in[s]$ with $\{i j\},\{i k\} \in E(\Gamma)$ it follows that $\{j k\} \in E(\Gamma)$. In fact the triangle property says that it is possible to find a "good" main prime in the terminology of [8, Example 4.3], which we recall next.

Example 2.2. Let $n=10, P_{1}=\left(x_{1}, \ldots, x_{7}\right), P_{2}=\left(x_{3}, \ldots, x_{8}\right), P_{3}=\left(x_{1}, \ldots, x_{4}\right.$, $\left.x_{8}, \ldots, x_{10}\right), P_{4}=\left(x_{1}, x_{2}, x_{5}, x_{8}, x_{9}, x_{10}\right), P_{5}=\left(x_{5}, \ldots, x_{10}\right)$ and $I=\bigcap_{i=1}^{5} P_{i}$. Then $q(I)=2$ and $\operatorname{depth}_{S} I=4$. The graph associated to $I$ on [5] as above has edges

$$
E(\Gamma)=\{\{13\},\{15\},\{35\},\{14\},\{23\},\{24\}\}
$$

and has the triangle property, but only $\{5\}$ is a "good" vertex; that is, for all $j, k \in[4]$ with $\{j 5\},\{k 5\} \in E(\Gamma)$ it follows that $\{j k\} \in E(\Gamma)$. Below you have the picture of $\Gamma$ :

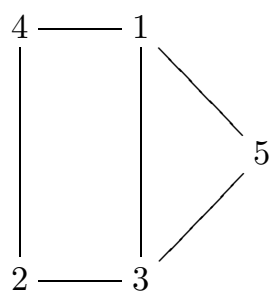

Proposition 2.3. If the bigsize of $I$ is two and $\Gamma=\Gamma(I)$ has the triangle property, then $\operatorname{sdepth}_{S} I \geq \operatorname{depth}_{S} I$.

Proof. Renumbering $\left\{P_{i}\right\}$ we may suppose that $i=1$; that is, for all $j, k \in[s]$ with $\{1 j\},\{1 k\} \in E(\Gamma)$ it follows that $\{j k\} \in E(\Gamma)$ by the triangle property. We repeat mostly the proof of Proposition [1.8. Applying [8, Theorem 1.6] for $\mathcal{F}$ containing some $\tau_{j}=\{j\}$, and $\tau_{j k}=\{j, k\}, 1<j, k \leq s, j \neq k$ we get $\operatorname{sdepth}_{S} I \geq$ $\left.\min \left\{A_{0},\left\{A_{\tau_{j}}\right\}_{\tau_{j} \in \mathcal{F}}\right\},\left\{A_{\tau_{j k}}\right\}_{\tau_{j k} \in \mathcal{F}}\right\}$. Note that the bigsize of $J_{\tau}$ is $\leq 1$ (similarly $\left.L_{\tau}\right), \tau \in \mathcal{F}$ and so $\operatorname{septh}_{S_{\tau}} J_{\tau} \geq \operatorname{depth}_{S_{\tau}} J_{\tau}$ by [8, Corollary 1.7]. If $P_{1}+P_{j} \neq m$, then

$A_{\tau_{j}} \geq \operatorname{sdepth}_{S_{\tau_{j}}} J_{\tau_{j}}+\operatorname{sdepth}_{S^{\prime}}\left(P_{j} \cap S^{\prime}\right) \geq 1+\operatorname{dim} S /\left(P_{1}+P_{j}\right)+\left\lceil\frac{\operatorname{height}\left(P_{j} \cap S^{\prime}\right)}{2}\right\rceil$.

Thus $A_{\tau_{j}} \geq 2+q \geq \operatorname{depth}_{S} I$ by Lemmas 1.4. 1.5. If $P_{1}+P_{j}=m$ but there exists $e \neq j$ such that $P_{e}+P_{j} \neq m$, then $\operatorname{sdepth}_{S_{\tau_{j}}} J_{\tau_{j}} \geq \operatorname{depth}_{S_{\tau_{j}}} J_{\tau_{j}}=1+$ $\operatorname{depth}_{S} S /\left(\bigcap_{u \neq j}\left(P_{u}+P_{j}\right)\right) \geq 1+q$, and so again $A_{\tau_{j}} \geq 2+q \geq \operatorname{depth}_{S} I$. If $P_{e}+P_{j}=m$ for all $e \neq j$, then $\operatorname{depth}_{S} I=2$ by [8, Lemma 1.3] and clearly $A_{\tau_{j}} \geq \operatorname{depth}_{S} I$. 
Now note that if $P_{1}+P_{j} \neq m \neq P_{1}+P_{k}, P_{j}+P_{k} \neq m$, then

$$
A_{\tau_{j k}} \geq \operatorname{sdepth}_{S_{\tau_{j k}}} J_{\tau_{j k}}+\operatorname{sdepth}_{S^{\prime}} L_{\tau_{j k}} \geq 1+\operatorname{sdepth}_{S^{\prime}}\left(P_{j} \cap P_{k} \cap S^{\prime}\right) \geq \operatorname{depth}_{S} I
$$

by Proposition 1.6. If $P_{1}+P_{j}=m, P_{j}+P_{k} \neq m$, then $P_{1}+P_{k} \neq m$ by the triangle property and $\operatorname{sdepth}_{S^{\prime}} L_{\tau_{j k}} \geq \operatorname{depth}_{S^{\prime}} L_{\tau_{j k}}=1+\operatorname{dim} S /\left(P_{1}+P_{k}\right) \geq 1+q$. Thus $A_{\tau_{j k}} \geq 2+q \geq \operatorname{depth}_{S} I$. If $I \cap S^{\prime \prime} \neq 0$, then as in the proof of Proposition 1.8. $A_{0} \geq \operatorname{depth}_{S} I$. Hence $\operatorname{sdepth}_{S} I \geq \operatorname{depth}_{S} I$.

Definition 2.4. The graph $\Gamma$ is a join graph if it is a join of two of its subgraphs; that is, after a renumbering of the vertices there exists $1 \leq c<s$ such that $\{i j\} \in$ $E(\Gamma)$ for all $1 \leq i \leq c, c<j \leq s$. Thus in Lemmas 1.4 1.5 one may say that $\operatorname{depth}_{S} S / I=1$ if and only if the associated graph of $I$ is a join graph. Let $\Gamma_{1}, \Gamma_{2}$ be graphs on $[r]$, respectively $\{r, r+1, \ldots, s\}$, for some integers $1<r \leq s-2$. Let $\Gamma$ be the graph on $[s]$ given by $E(\Gamma)=E\left(\Gamma_{1}\right) \cup E\left(\Gamma_{2}\right) \cup\{\{i j\}: 1 \leq i<r, r<j \leq s\}$. We call $\Gamma$ the graph given by concatenation of $\Gamma_{1}, \Gamma_{2}$ in the vertex $\{r\}$.

Lemma 2.5. Let $I=\bigcap_{i=1}^{s} P_{i}$ be the intersection of the minimal monomial prime ideals of $S / I, I_{1}=\bigcap_{i=1}^{r} P_{i}, I_{2}=\bigcap_{i \geq r}^{s} P_{i}$ and $\Gamma, \Gamma_{1}, \Gamma_{2}$ be the graphs associated to $I$, respectively $I_{1}, I_{2}$ as in the previous section. Suppose that $\sum_{i=1}^{s} P_{i}=m, \Gamma$ is the concatenation of $\Gamma_{1}, \Gamma_{2}$ in $\{r\}$ and bigsize $(I)=2$. Then

$$
\operatorname{depth}_{S} I=\min \left\{\operatorname{depth}_{S} I_{1}, \operatorname{depth}_{S} I_{2}\right\} .
$$

Proof. We consider the following exact sequence:

$$
0 \rightarrow S / I \rightarrow S / I_{1} \oplus S / I_{2} \rightarrow S / I_{1}+I_{2} \rightarrow 0 .
$$

Since $P_{i}+P_{j}=m$ for all $1 \leq i<r, r<j \leq s$, we get $I_{1}+I_{2}=P_{r}$. But $\operatorname{depth}_{S} S / I, \operatorname{depth}_{S} S / I_{i} \leq \operatorname{depth}_{S} S / P_{r}$ for $i=1,2$ and by the Depth Lemma 12 . we get

$$
\operatorname{depth}_{S} S / I=\min \left\{\operatorname{depth}_{S} S / I_{1}, \operatorname{depth}_{S} S / I_{2}\right\} .
$$

Remark 2.6. Let $I=\bigcap_{i=1}^{3} P_{i}$ be the intersection of the minimal monomial prime ideals of $S / I$. Suppose that $P_{1}+P_{2} \neq m \neq P_{1}+P_{3}$ and $P_{2}+P_{3}=m$. Let $I_{1}=$ $P_{1} \cap P_{2}, I_{2}=P_{1} \cap P_{3}$ and $\Gamma, \Gamma_{1}, \Gamma_{2}$ be the graphs associated to $I$, respectively $I_{1}$, $I_{2}$. We have $E\left(\Gamma_{1}\right)=E\left(\Gamma_{2}\right)=\emptyset$ and $E(\Gamma)=\{\{23\}\}$. Then $\Gamma$ is the concatenation of $\Gamma_{1}, \Gamma_{2}$ in $\{1\}$ and

$\operatorname{depth}_{S} I=\min \left\{\operatorname{depth}_{S} I_{1}, \operatorname{depth}_{S} I_{2}\right\}=2+\min \left\{\operatorname{dim} S /\left(P_{1}+P_{2}\right), \operatorname{dim} S /\left(P_{1}+P_{3}\right)\right\}$ by the above lemma. That is the formula found in [5, Proposition 2.1].

Proposition 2.7. Let $I=\bigcap_{i=1}^{s} P_{i}$ be the intersection of the minimal monomial prime ideals of $S / I$ and $\Gamma$ be the graph associated to $I$. Suppose that $\sum_{i=1}^{s} P_{i}=m$, bigsize $(I)=2$ and $\operatorname{depth}_{S} I>3$. Then after renumbering $\left\{P_{i}\right\}$ there exists $1<$ $r \leq s-2$ such that $\Gamma$ is the concatenation in $\{r\}$ of the graphs $\Gamma_{1}, \Gamma_{2}$ associated to $I_{1}=\bigcap_{i=1}^{r} P_{i}$, respectively $I_{2}=\bigcap_{i \geq r}^{s} P_{i}$. Moreover, $\operatorname{depth}_{S} I_{1}, \operatorname{depth}_{S} I_{2}>3$.

Proof. Since bigsize $(I)=2$ we may suppose that $P_{s-1}+P_{s} \neq m$, that is, $\{s-1, s\} \notin$ $\Gamma$. Consider the following exact sequence:

$$
\begin{aligned}
0 \rightarrow S / I \rightarrow S / P_{1} \cap \ldots \cap P_{s-1} \oplus S / P_{1} \cap \ldots \cap P_{s-2} \cap P_{s} \\
\rightarrow S / P_{1} \cap \ldots \cap P_{s-2} \cap\left(P_{s}+P_{s-1}\right) \rightarrow 0 .
\end{aligned}
$$

As in the proof of Proposition 2.3 we see that $P_{i} \not \subset P_{s}+P_{s-1}$ for $i<s-1$ because bigsize $(I)=2$. Then $\operatorname{depth}_{S} S / P_{1} \cap \ldots \cap P_{s-2} \cap\left(P_{s}+P_{s-1}\right)=1$ using 
[8, Corollary 1.7]. By the Depth Lemma we get, let us say, $\operatorname{depth}_{S} S / P_{1} \cap \ldots \cap P_{s-1}=$ 1 since $\operatorname{depth}_{S} S / I>2$. After a renumbering of $\left\{P_{i}\right\}_{i<s-1}$ using Lemmas 1.4, 1.5. we may suppose that there exists $1 \leq c<s-1$ such that $P_{i}+P_{j}=m$ for all $1 \leq i \leq c, c<j<s$. Set $r=c+1$ and renumber $P_{s}$ by $P_{r}$ and $P_{i}$ by $P_{i+1}$ for $r \leq i<s$. Then $I_{1}=\bigcap_{i=1}^{r} P_{i}$ and $I_{2}=\bigcap_{i \geq r}^{s} P_{i}$ satisfy our proposition. The last statement follows by Lemma 2.5 .

Corollary 2.8. Let $I=\bigcap_{i=1}^{s} P_{i}$ be the intersection of the minimal monomial prime ideals of $S / I$. Suppose that $\sum_{i=1}^{s} P_{i}=m$, bigsize $(I)=2$ and $q(I)>1$. Then depth $_{S} I=2+q(I)>3$ if and only if the graph associated to $I$ is a concatenation of several graphs on two vertices having no edges.

Proof. The necessity follows by applying the above proposition by recurrence and the sufficiency follows by applying Lemma 2.5 by recurrence.

Corollary 2.9. Let $I=\bigcap_{i=1}^{s} P_{i}, I^{\prime}=\bigcap_{i=1}^{s} P_{i}^{\prime}$ be the intersection of the minimal monomial prime ideals of $S / I$, respectively $S / I^{\prime}$. Suppose that $\sum_{i=1}^{s} P_{i}=m=$ $\sum_{i=1}^{s} P_{i}^{\prime}$, bigsize $(I)=$ bigsize $\left(I^{\prime}\right)=2$ and $q(I)=q\left(I^{\prime}\right)$. If the graphs associated to $I$, respectively $I^{\prime}$, coincide, then $\operatorname{depth}_{S} I=\operatorname{depth}_{S} I^{\prime}$.

Proof. By the above corollary, $\operatorname{depth}_{S} I>3$ and depth ${ }_{S} I^{\prime}>3$ hold if and only if the graphs $\Gamma(I), \Gamma\left(I^{\prime}\right)$ are concatenations of several graphs on two vertices having no edges. Since $\Gamma(I)=\Gamma\left(I^{\prime}\right)$ we get that $\operatorname{depth}_{S} I>3$ if and only if $\operatorname{depth}_{S} I^{\prime}>3$. But Lemmas 1.4 1.5 say that in this case $\operatorname{depth}_{S} I=2+q(I)=2+q\left(I^{\prime}\right)=$ $\operatorname{depth}_{S} I^{\prime}$. Note that $\operatorname{depth}_{S} I=2$ holds if and only if $\Gamma(I)=\Gamma\left(I^{\prime}\right)$ is a join graph which happens if and only if $\operatorname{depth}_{S} I^{\prime}=2$. Then necessarily $\operatorname{depth}_{S} I=\operatorname{depth}_{S} I^{\prime}$ also in the remaining case $\operatorname{depth}_{S} I=3$.

Theorem 2.10. Let the depth of a monomial squarefree ideal I of $S$ be such that the sum of every three different ideals of its minimal prime ideals is the maximal ideal of $S$, or more generally a constant ideal of $S$. Then the depth of I does not depend on the characteristic of $K$.

Proof. It is enough to suppose that $\sum_{i=1}^{s} P_{i}=m$ and bigsize $(I)=2$, size $(I)=1$ by Corollary 1.7. By Lemmas 1.4, 1.5] (see also Remark 2.4), $\operatorname{depth}_{S} I=2$ if and only if the graph $\Gamma(I)$ associated to $I$ is a join graph which is a combinatorial characterization and so does not depend on $p=$ char $K$. By Corollary 2.8, $\operatorname{depth}_{S} I=2+q(I)>3$ if and only if $q(I)>1$ and $\Gamma(I)$ is a concatenation of several graphs on two vertices having no edges, the exact value of $\operatorname{depth}_{S} I$ being given by $q(I)$. Thus again $\operatorname{depth}_{S} I$ does not depend on $p$. Finally, $\operatorname{depth}_{S} I=3$ happens only when we are not in the above cases; that is, it does not depend on $p$.

Remark 2.11. The above theorem fails if just the sum of every six minimal prime ideals of $I$ is the maximal ideal of $S$. The Stanley-Reisner ideal $I$ of the simplicial complex associated to the canonical triangulation of the real projective plane has bigsize $(I)=5$, size $(I)=2$ and $\operatorname{depth}_{S} I=4$ if char $K \neq 2$; otherwise $\operatorname{depth}_{S} I=3$. 


\section{Stanley's Conjecture for monomial squarefree ideals OF BIGSIZE 2}

The case when $\operatorname{depth}_{S} I>1+$ bigsize $(I)$ is unusually big, and it is hard to check Stanley's Conjecture in this case. Next we will construct such examples, where Lemma 2.5 and Proposition 2.7 prove to be very useful.

Example 3.1. Let $\Gamma_{1}$ be the graph given on $\{1,2,5\}$ by $E\left(\Gamma_{1}\right)=\{\{15\}\}$ and let $\Gamma_{2}$ be the graph given on $\{3,4,5\}$ by $E\left(\Gamma_{1}\right)=\{\{35\}\}$. Suppose that $I_{1}=P_{1} \cap P_{2} \cap P_{5}$ and $I_{2}=P_{3} \cap P_{4} \cap P_{5}$ are irredundant intersections of monomial prime ideals of $S$ with $q\left(I_{1}\right)>1, q\left(I_{2}\right)>1$, bigsize $\left(I_{1}\right)=\operatorname{bigsize}\left(I_{2}\right)=2$. Then $\operatorname{depth}_{S} I=$ $2+\min \left\{\operatorname{dim} S / P_{1}+P_{2}, \operatorname{dim} S / P_{2}+P_{5}\right\} \geq 2+q\left(I_{1}\right)>3$ by [5] (see Remark 2.6]) if $q\left(I_{1}\right)>1$. Similarly, $\Gamma_{2}$ is the graph associated to $I_{2}$ and $\operatorname{depth}_{S} I_{2}>3$. Let $\Gamma$ be the concatenation in $\{5\}$ of $\Gamma_{1}$ and $\Gamma_{2}$. If $I=I_{1} \cap I_{2}$ is an irredundant intersection of those 5-prime ideals and $q(I)>1$, $\operatorname{bigsize}(I)=2$, then $\Gamma$ is the graph associated to $I$ and $\operatorname{depth}_{S} I>3$ by Lemma 2.5. This is the graph from Example 2.2.

The above example is not bad because this case can be handled by our Proposition 2.3 that is, there exists a "good" main prime $P_{5}$. Are there ideals $I$ for which there exists no "good" main prime, that is, for which the graph associated to $I$ does not have the triangle property? Next we will construct such a bad example. First we will see how its graph should look.

Example 3.2. Let $\Gamma_{1}$ be the graph constructed above on [5] and $\Gamma_{2}$ be the graph given on $\{1,6\}$ with $E\left(\Gamma_{2}\right)=\emptyset$. Let $\Gamma$ be the concatenation in $\{1\}$ of $\Gamma_{1}$ and $\Gamma_{2}$. Below you have the picture of $\Gamma$, and clearly it does not satisfy the triangle property. If we show that $\Gamma$ is the graph associated to a monomial squarefree ideal $I$ of $S$ with bigsize $(I)=2$ and $q(I)>1$, then we will have $\operatorname{depth}_{S} I>3$ by Lemma 2.5. This is done in the next example.

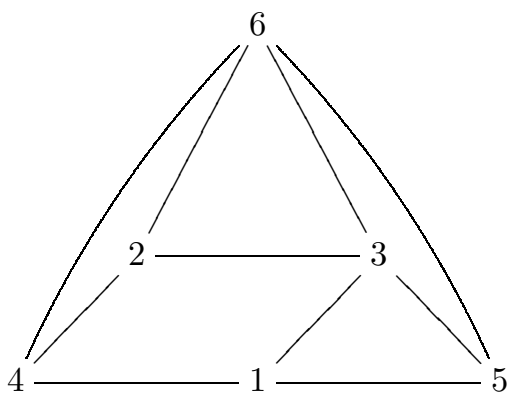

Example 3.3. Let $n=12, P_{1}=\left(x_{1}, x_{4}, x_{5}, x_{6}, x_{9}, \ldots, x_{12}\right), P_{2}=\left(x_{1}, x_{4}, \ldots, x_{10}\right)$, $P_{3}=\left(x_{1}, x_{2}, x_{3}, x_{7}, x_{8}, \ldots, x_{12}\right), P_{4}=\left(x_{1}, x_{2}, x_{3}, x_{6}, x_{7}, x_{8}, x_{11}, x_{12}\right), P_{5}=$ $\left(x_{1}, \ldots, x_{8}\right), P_{6}=\left(x_{2}, \ldots, x_{6}, x_{9}, \ldots, x_{12}\right)$ and $I=\bigcap_{i=1}^{6} P_{i}$. We have $P_{1}+P_{4}=$ $P_{1}+P_{5}=P_{1}+P_{3}=P_{2}+P_{3}=P_{2}+P_{4}=P_{2}+P_{6}=P_{3}+P_{5}=P_{3}+P_{6}=$ $P_{4}+P_{6}=P_{5}+P_{6}=m$ and $P_{1}+P_{2}=m \backslash\left\{x_{2}, x_{3}\right\}, P_{1}+P_{6}=m \backslash\left\{x_{7}, x_{8}\right\}$, $P_{2}+P_{5}=m \backslash\left\{x_{11}, x_{12}\right\}, P_{3}+P_{4}=m \backslash\left\{x_{4}, x_{5}\right\}, P_{4}+P_{5}=m \backslash\left\{x_{9}, x_{10}\right\}$. Clearly, bigsize $(I)=2=q(I)$ and the graph $\Gamma$ associated to $I$ is the graph constructed in Example 3.2. We have $\operatorname{depth}_{S} I=2+q(I)=4$ by Lemma 2.5. Let $S^{\prime}=K\left[x_{2}, \ldots, x_{12}\right]$ and $P_{i}^{\prime}=P_{i} \cap S^{\prime}, I^{\prime}=I \cap S^{\prime}$. We have $I^{\prime}=\bigcap_{i=1}^{5} P_{i}^{\prime}$ because $P_{1}^{\prime} \subset P_{6}^{\prime}$. The graph associated to $I^{\prime}$ is in fact $\Gamma_{1}$ from the above example and has 
the triangle property. Then by Proposition 2.3 we get $\operatorname{sdepth}_{S^{\prime}} I^{\prime} \geq \operatorname{depth}_{S^{\prime}} I^{\prime}=$ $2+q\left(I^{\prime}\right)=4$ because $q\left(I^{\prime}\right)=q(I)$. Using the decomposition $I=I^{\prime} \oplus x_{1}\left(I: x_{1}\right)$ as linear spaces we get

$$
\begin{gathered}
\operatorname{sdepth}_{S} I \geq \min \left\{\operatorname{sdepth}_{S^{\prime}} I^{\prime}, \operatorname{sdepth}_{S}\left(I: x_{1}\right)\right\} \geq \min \left\{4, \operatorname{sdepth}_{S} P_{6}\right\} \\
=\min \left\{4,\left\lceil\frac{9}{2}\right\rceil+3\right\}=4=\operatorname{depth}_{S} I .
\end{gathered}
$$

This gives us the idea to handle such bad examples in the next proposition.

Proposition 3.4. Let $I=\bigcap_{i=1}^{s} P_{i}$ be the intersection of the minimal monomial prime ideals of $S / I$. Suppose that $\sum_{i=1}^{s} P_{i}=m$, bigsize $(I)=2$ and $\operatorname{depth}_{S} I>3$. Then $\operatorname{sdepth}_{S} I \geq \operatorname{depth}_{S} I$.

Proof. Apply induction on $s$. The cases $s \leq 4$ are given in [9], [5], 8]. Assume that $s>4$ and let $\Gamma$ be the graph of $I$. By Proposition 2.7 we may suppose after renumbering $\left(P_{i}\right)$ that there exists $1<r \leq s-2$ such that $\Gamma$ is the concatenation in $\{r\}$ of the graphs $\Gamma_{1}, \Gamma_{2}$ associated to $I_{1}=\bigcap_{i=1}^{r} P_{i}$, respectively $I_{2}=\bigcap_{i=r}^{s} P_{i}$. Note that if $r=2$ or $s-r=2$, then bigsize $\left(I_{i}\right)$ could not be 2 , but this causes no trouble since we need the bigsize 2 only to apply to Proposition 2.7. From Lemma 2.5 it follows that $\operatorname{depth}_{S} I=\min \left\{\operatorname{depth}_{S} I_{1}, \operatorname{depth}_{S} I_{2}\right\}$ and so $\operatorname{depth}_{S} I_{i}>3$ for $i=1,2$. Note that $P_{r} \backslash P_{j} \subset P_{i} \backslash P_{j}=m \backslash P_{j}$ for all $1 \leq i<r, r<j \leq s$. After renumbering variables we may suppose that $\left\{x_{1}, \ldots, x_{e}\right\}, 1 \leq e<n$, are all variables of $\bigcup_{j>r}^{s}\left(P_{r} \backslash P_{j}\right)$. As we noticed they are contained in any $P_{i}, 1 \leq i<r$. Set $S^{\prime}=K\left[x_{e+1}, \ldots, x_{n}\right], P_{i}^{\prime}=P_{i} \cap S^{\prime}, I^{\prime}=I \cap S^{\prime}$. Then $P_{r}^{\prime} \subset P_{j}^{\prime}$ for all $r<j \leq s$, and we get $I^{\prime}=\bigcap_{i=1}^{r} P_{i}^{\prime}$. Moreover, since $\left\{x_{1}, \ldots, x_{e}\right\}$ is contained in any $P_{i}, 1 \leq i \leq r$ we see that the "relations" between these prime ideals are preserved after intersection with $S^{\prime}$ and the graph $\Gamma^{\prime}$ of $I^{\prime}$ is in fact $\Gamma_{1}$. Moreover, $q\left(I^{\prime}\right)=q\left(I_{1}\right)$ and bigsize $\left(I^{\prime}\right)=\operatorname{bigsize}\left(I_{1}\right)$. Then $\operatorname{depth}_{S^{\prime}} I^{\prime}=\operatorname{depth}_{S} I_{1}$ by Corollary [2.9, the case $r=2$ being trivial. Using the induction hypothesis on $s$ we get $\operatorname{sdepth}_{S^{\prime}} I^{\prime} \geq \operatorname{depth}_{S^{\prime}} I^{\prime}$. We have the decomposition $I=I^{\prime} \oplus\left(\left(x_{1}, \ldots, x_{e}\right) \cap I\right)$ as linear spaces, and it follows that

$$
\operatorname{sdepth}_{S} I \geq \min \left\{\operatorname{sdepth}_{S^{\prime}} I^{\prime}, \operatorname{sdepth}_{S}\left(\left(x_{1}, \ldots, x_{e}\right) \cap I\right)\right\} .
$$

But $J=\left(x_{1}, \ldots, x_{e}\right) \cap I=\bigcap_{i>r}^{s} P_{i}^{\prime} \cap\left(x_{1}, \ldots, x_{e}\right)$ because $\left(x_{1}, \ldots, x_{e}\right) \subset P_{i}$ for $1 \leq i \leq r$, and the decomposition is irredundant since if $\left(x_{1}, \ldots, x_{e}\right) \subset P_{j}$, then $P_{r} \subset P_{j}$, which is false. Note that $q(J)=q\left(I_{2}\right)$ and the graph associated to $J$ coincides with $\Gamma_{2}$. Again by Corollary 2.9. $\operatorname{depth}_{S} J=\operatorname{depth}_{S} I_{2}>3$, the case $r=2$ being trivial. Using the induction hypothesis on $s$ we get $\operatorname{sdepth}_{S} J \geq$ $\operatorname{depth}_{S} J$, and so

$\operatorname{sdepth}_{S} I \geq \min \left\{\operatorname{sdepth}_{S^{\prime}} I^{\prime}, \operatorname{sdepth}_{S} J\right\} \geq \min \left\{\operatorname{depth}_{S} I_{1}, \operatorname{depth}_{S} I_{2}\right\}=\operatorname{depth}_{S} I$.

Theorem 3.5. Let $I$ be a monomial squarefree ideal of $S$ such that the sum of every three different of its minimal prime ideals is the maximal ideal of $S$, or more generally a constant ideal $J$ of $S$. Then $\operatorname{sdepth}_{S} I \geq \operatorname{depth}_{S} I$.

The proof follows from Theorem 1.8 and the above proposition, the reduction to the case $J=m$ being given by [1, Lemma 3.6]. 


\section{REFERENCES}

[1] J. Herzog, M. Vladoiu, X. Zheng, How to compute the Stanley depth of a monomial ideal, J. Algebra, 322 (2009), 3151-3169. MR2567414 (2010k:13036)

[2] J. Herzog, D. Popescu, M. Vladoiu, Stanley depth and size of a monomial ideal, Proc. Amer. Math. Soc. 140 (2012), 493-504.

[3] M. Ishaq, Upper bounds for the Stanley depth, to appear in Comm. Algebra, arXiv:AC/1003.3471.

[4] G. Lyubeznik, On the arithmetic rank of monomial ideals. J. Algebra, 112 (1988), 86-89. MR.921965 (89b:13020)

[5] A. Popescu, Special Stanley Decompositions, Bull. Math. Soc. Sc. Math. Roumanie, 53(101), no. 4 (2010), 363-372. MR2777680

[6] D. Popescu, Stanley depth of multigraded modules, J. Algebra, 321 (2009), 2782-2797. MR2512626 (2010f:13007)

[7] D. Popescu, An inequality between depth and Stanley depth, Bull. Math. Soc. Sc. Math. Roumanie, 52(100) (2009), 377-382. MR2554495 (2010h:13019)

[8] D. Popescu, Stanley conjecture on intersections of four monomial prime ideals. Submitted to Comm. Algebra.

[9] D. Popescu, I. Qureshi, Computing the Stanley depth, J. Algebra, 323 (2010), 2943-2959. MR2609185(2011i:13024)

[10] A. Rauf, Depth and Stanley depth of multigraded modules, Comm. Algebra, 38 (2010), 773784. MR2598911 (2011g:13029)

[11] R. P. Stanley, Linear Diophantine equations and local cohomology, Invent. Math., 68 (1982), 175-193. MR666158 (83m:10017)

[12] R. H. Villarreal, Monomial Algebras, Marcel Dekker Inc., New York, 2001. MR1800904 (2002c:13001)

Institute of Mathematics "Simion Stoilow", University of Bucharest, P.O. Box 1-764, Bucharest 014700, Romania

E-mail address: dorin.popescu@imar.ro 